\title{
News
}

\section{Making Time}

\author{
Kyle Jewer \\ 'Class of 2013, Faculty of Medicine, Dalhousie University
}

It is possible to store the mind with a million facts and still be entirely uneducated. Aleck William Bourne, British gynaecologist

W hen it comes to volunteering, medical students are stuck in a vexing paradox. We are presented with an unrivaled breadth of opportunities and resources, as we have increased access to funding, and to motivated faculty members and administrators, who provide guidance and connections. Most importantly, however, we are surrounded by a diversely talented group of peers with whom we can collaborate. Without question, we are well positioned to organize ventures that can positively impact our community. Despite all this, extra-curricular activities are often the first sacrifice made to pare down an increasingly busy schedule. The demands of medical school can be daunting and, at times, overwhelming, even the most organized individuals.

Medical education programs in Canada are time-tested and taught by committed clinician-educators with a vested interest in helping us rise to our maximum potential. Much of what we require to become knowledgeable physicians is provided to us as part of these programs. Of course, many of these resources are only as valuable as our desire to use them. We maximize the value of our medical training by tailoring it to our own interests; no education is 'one size fits all.' We all do this by choosing elective experiences in the fields we wish to pursue and by forging bonds with mentors whose clinical philosophies we wish to emulate in our own practice.

An element of our education that is often abandoned upon entry into medical school is volunteerism, be it on a local or global level. Certainly our training includes countless hours of patient contact, and the interpersonal demands of clinical learning may feel like interaction enough. On the other hand, we will be spending our careers as integral members of a society that is more socially complex than we may realize. Sadly, underserved and underprivileged demographics have been shown to have poor health outcomes and will continue to make up a significant proportion of health care users. Arguably, one such population is the special needs community. Despite the disproportionately high prevalence of developmental and behavioural disorders in paediatric patients, interaction with persons with special needs is strikingly absent from our pre-clerkship program. For many of us, interacting with these individuals makes up a small fraction of our daily social experiences, if any at all. In many cases, they face challenges foreign to the average medical student. To best serve these individuals we must first understand those challenges. The best way to do this is to seek out opportunities to engage with them firsthand.

To improve our understanding of this important segment of patients, a classmate, Rachel Shaw and I developed a program in which student volunteers teach one-on-one swimming lessons to children with special needs. At the outset the benefits were clear and reciprocal: by working together in the pool, the students stood to develop their communication skills and the children had the opportunity to learn how to swim. As the weeks went by, however, greater triumphs were realized: a shy autistic child made eye contact with his instructor for the first time and another overcame his fear of separation and left his parents to get in the pool with his instructor. To the untrained eye these events would seem trivial, but to the instructors the importance of the progress was clear.

By the end of our eight-week session, it was apparent that the children not only benefited from the actual swimming lessons, but also developed social skills and trust. Likewise, the experience not only enhanced the volunteers' ability to interact with their child, but also fostered an understanding of the obstacles faced by children with special needs and their families.

For me, the clearest view of this diverse group came by the side of the pool, when I took a moment to stand 
back and appreciate the scene in front of me. While their children, a wild torrent of water and bright bathing suits, squealed and splashed in the water, the parents surrounded the pool, chatting familiarly. Discussions of progress at home and at school, mutual friends and neighbours, shared experiences, and helpful advice abound in this collegial group. Though physically dispersed across the city, they form a tightly knit community of allies, whose friendships are based on shared struggles and successes.

It is important to not think of volunteering as a means to an end, be it getting into medical school or filling in a line on a CaRMS application. Much can be realized when time is invested in turning the resources at our disposal into assistance for those around us in need. It isn't until we immerse ourselves in our community that we truly appreciate its complexity and how valuable this work really can be.
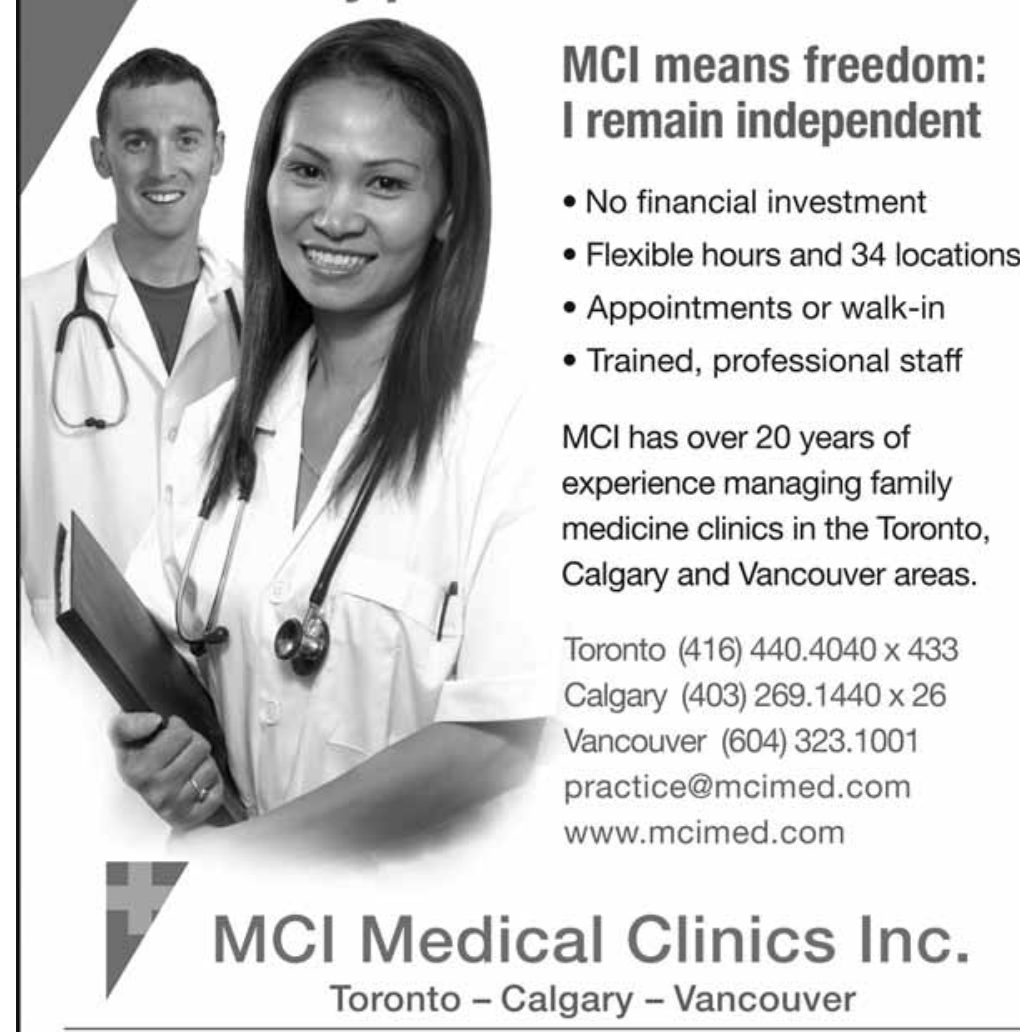

Works for Doctors - Cares for Patients - Empowers Employees

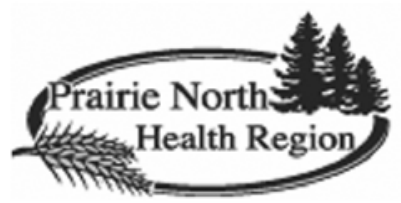

Opportunities for Family Physicians

FAMILY PHYSICIAN

\section{EMERGENCY DEPARTMENT PHYSICIAN PSYCHIATRIST}

\section{South West Health \\ Working Together for Better Health \\ Shelburne · Yarmouth · Digby}

Are you seeking a healthy work/life balance or are you interested in maximizing your income. If so, we have the opportunity for you ........

- Fee-for-service practices allow you to combine family, recreation and a fulfilling medical practice or maximize your income with a full spectrum practice.

- Turn-key community health centres with full EMR available.

- Ask about our generous signing bonuses.

- Assistance with relocation expenses.

\section{Our Communities offer....}

- Abundance of rivers, lakes and ocean for water recreation

- White sand beaches dot our coast

- Safe, friendly, amazing life style

- Rich French Acadian culture

- Ferry service to New Brunswick

- Air travel to USA

To learn more about South West Health and our communities, go to: http://www.swndha.nshealth.ca/recruitbroch.pdf and download our recruitment brochure.

\section{Contact:}

\section{Shirley Watson-Poole}

Physician Resource Manager

South West Health

60 Vancouver Street

Yarmouth, NS B5A 2P5

Phone: (902) 742-3542 ext. $451 \quad$ Fax: (902) 742-0369

Email: swatson@swndha.nshealth.ca
The Prairie North Health Region of Saskatchewan currently has practice opportunities for Family Physicians in the following communities:

Meadow Lake, SK. has opportunities for family physicians in locum or permanent, full or part-time positions. Applicants would be joining an established clinic practice which provides a full scope of services including attendance at Meadow Lake Hospital; a 30 bed facility providing acute and emergency care. The clinic has recently become a Primary Care Facility and is currently working with the College of Medicine, University of Saskatchewan, to become a Rural site for distributed medical education this reason, preference will be given to CCFP qualified applicants with an interest in supporting both the service and academic mission of the community. Meadow Lake is a community of 5,000+ in the North-east corner of the Region but provides medical services to an extended population in surrounding smaller communities both within the Region and adjacent Health Regions.

Lloydminster, SK. is a community of 26,000 straddling the western boundary of the Region and extending into Alberta. Full or part-time, locum or permanent positions are available in established clinics in a fee-for-service environment. Lloydminster is the site of a 66 bed Regional hospital attended by both family physicians and specialists in Surgery, Internal Medicine, Obstetrics and Gynecology and Psychiatry. It provides service to an extended population of both Alberta and Saskatchewan residents. Provision of Obstetrical Services is optional, and there is a possibility of participation in Emergency Room services.

The communities of Maidstone and Turtleford, SK also have opportunities for physicians to join small group, practice providing services to these and surrounding communities as well as emergency and inpatient coverage to the Community Hospitals in Maidstone and Turtleford. The practices in these communities are supported by an alternate payment model.

For all of these opportunities there are provincial, community and Regional incentive programs. Expressions of interest and Curricula vitae may be submitted to: and be a permanent location for both undergraduate and postgraduate training. For 